\title{
Timelessness and Time Dependence of Human Consciousness From a Scientific Western Viewpoint
}

\author{
Franz Klaus Jansen \\ Independent Researcher
}

\begin{abstract}
Eastern philosophy and western science have convergent and divergent viewpoints for their explanation of consciousness. Convergence is found for the practice of meditation allowing besides a time dependent consciousness, the experience of a timeless consciousness and its beneficial effect on psychological wellbeing and medical improvements, which are confirmed by multiple scientific publications. Theories of quantum mechanics with non-locality and timelessness also show astonishing correlation to eastern philosophy, such as the theory of Penrose-Hameroff (ORC-OR), which explains consciousness by reduction of quantum superposition in the brain. Divergence appears in the interpretation of the subjective experience of timeless consciousness. In eastern philosophy, meditation at a higher level of awareness allows the personal experience of timeless and non-dual consciousness, considered as an empirical proof for the existence of pure consciousness or spirituality existing before the material world and creating it by design. Western science acknowledges the subjective, non-dual experience, and its multiple beneficial effects, however, the interpretation of spirituality designing the material universe is in disagreement with the Darwinian Theory of mutation and selection. A design should create an ideal universe without the injustice of $3 \%$ congenital birth defects and later genetic health problems. The western viewpoint of selection is more adapted to explain congenital errors. The gap between subjectivity and objectivity, the mind-body problem, is in eastern philosophy reduced to the dominance of subjectivity over objectivity, whereas western science attributes equal values to both. Nevertheless, there remains an astonishing complementarity between eastern and western practices.
\end{abstract}

Keywords: consciousness, meditation, timelessness, time dependence, quantum mechanics, eastern philosophy, western philosophy of science

\section{Introduction}

In western countries, awareness can be perceived as full awareness during the day, and partial awareness when waking up in the morning and lacking awareness during deep sleep. The Oxford dictionary defines awareness as "knowledge or perception of a situation or fact," which resembles a first person view of introspection. Consciousness is defined by a third person view "The state of being aware of and responsive to one's surroundings." In medicine, anaesthetics allow inducing an unconscious state in order to prevent the feeling of pain, which requires the verification, whether a patient is still conscious or not. The simplest way of testing the patient's consciousness is by asking him some questions, which corresponds to the definition in the

Franz Klaus Jansen, MD, Ph.D., Biological Research in Immunology at University of Düsseldorf, Germany and SANOFI, Pharmaceutical Industry, France; main research field: Philosophy of Science. Email: jansen.franz@ orange.fr. 
Oxford Dictionary.

Nevertheless, consciousness has over 40 different definitions (Vimal 2009), including first person and third person views. Eastern philosophy (Rao 2005) essentially considers the subjective first person aspect of consciousness, whereas western science is more based on the objective third person view of consciousness. The convergent and divergent view points between eastern philosophy and western science are analysed in more details.

\section{Convergent Eastern and Western Viewpoints on Consciousness}

Meditation is an ancient practice in Hinduism, already mentioned in the texts of Vedas, and consists in the training of the mind in order to become familiar with oneself (Wikipedia, Meditation). During profound meditation, the special subjective awareness of timeless consciousness is experienced. This practice has been introduced in western countries and is followed with great interest, since it has direct influence on personal wellbeing and also significant effects on medical and biological factors, as confirmed by many scientific publications. In addition, certain interpretations of quantum mechanics are in good agreement with the eastern viewpoint.

\subsection{Experience of Time Dependent and Timelessness Consciousness}

During an awaken conscious state, all sense organs are active and contribute to a mental representation of the exterior extra-mental environment and the interior body behaviour. With open eyes, there is direct physical contact between the extra-mental physical environment and its mental representation by physical electromagnetic waves entering the eye and activating neurones in the retina, which transmit their information to certain brain regions. Thereby, exterior objects find a representation in the brain. With open ears, similar contact is established between physical mechanical waves inducing vibrations of the tympanum in the ear, which activate the corresponding neurones and their transmission to a different brain region (Jansen 2014). Time characterised by duration with boundaries can also be considered as a perception, similar to seeing, hearing, and touching. The perception of time seems to be correlated to the amount of energy expended (Eagleman \& Pariyadath 2009). The boundaries of duration allow a comparison to other time consuming systems and can thereby be quantified. Conventional time boundaries, like those of a football game, can be compared to reference boundaries such as the revolution of the earth, defining 24 hours. In a similar way, all outside and inside sensory organs, like a heart strike, show duration and boundaries. This kind of conscious awareness with all sensory organs is time dependent.

In meditation at a higher awareness, timeless consciousness can be experienced. Although there are multiple practices, here a contemporary form of the Chopra Centre will be taken as example. Meditation requires the reduction of the activity of most sensory organs. Eyes have to be closed and no longer transmit the time boundaries imposed by outside events. Ears cannot be closed, but an environment with no noise or very calm music is chosen to reduce exterior disturbances. Not seeing and reduced hearing avoid the societal contact to other persons and help to concentrate on a person's subjective awareness. A fixed position without movements of all members facilitates inner concentration. Other disturbing influences, like weather conditions or uncomfortable temperatures, can also be avoided in adapted meditation rooms. Neutralisation of sense organ activities essentially eliminates the boundaries of time perception, imposed by sensory organs for extra-mental reality, thus, its duration becomes more and more extended until it appears as timeless. Deep meditation leads 
to a non-dual feeling of unicity, coherence, continuity, and selfhood (Rao 2005, 11), in which time boundaries have disappeared. This experience seems to be ideal for human life, since it liberates the person from the predicament of suffering and leads "the individual to higher states of awareness, achievement, and enjoyment" (Rao 2005, 10).
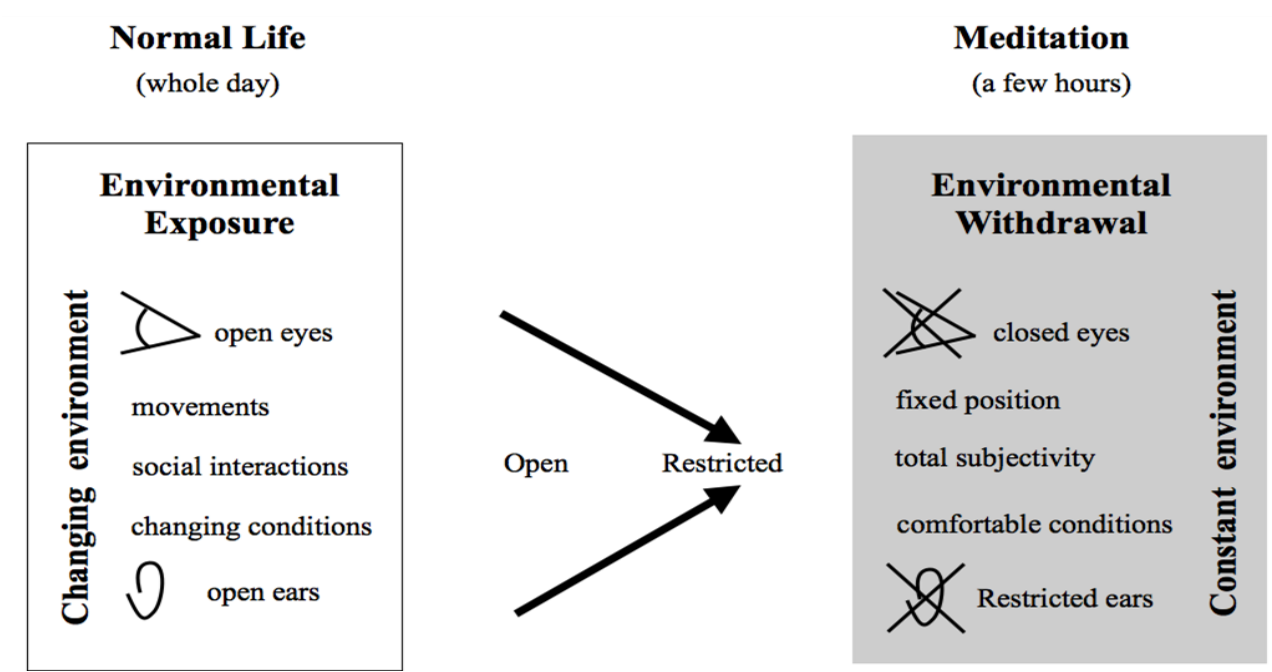

Fig. 1 Phenomenology of Consciousness.

The experience of consciousness in deep meditation requires the neutralization of many sense organs: left side before and right side during meditation.

After the end of mediation, all neutralised sense organs are re-activated again and once more impose their durations and boundaries. By reentering the duality environment of sense organs, the experienced timelessness of pure consciousness disappears.

The process of withdrawal from environmental duality to enter an inner timeless experience can be performed every day. The forth and back movements from time dependent active sense organs in normal life to their partial neutralisation for a timeless experience of consciousness in meditation and the reactivation of all sense organs to the environment again show the interdependence of an objective and a subjective world (Jansen 2011). The objective world allows the subjective world to exist and the subjective world diminishes major constraints like stress of the objective world. The experienced timelessness in meditation is the opposite of timelessness in physical formalism. During meditation, only the boundaries disappear for becoming timeless and duration is maintained, whereas in physics, duration is eliminated and its boundaries are maintained for the comparison with boundaries in other systems.

\subsection{Benefits of Meditation}

An abundant scientific literature shows that meditation practice increases psychological and biological wellbeing. There are positive correlations to objective neuro-scientific parameters as well as favourable evolutions during the treatment of several diseases and measurable changes in the expression of genes.

Neuro-scientific parameters are changed during meditation, such as an increase of the slower alpha and theta band power in electroencephalography. In imaging studies, the anterior cingulate cortex and the dorsolateral prefrontal cortex are activated (Cahn \& Polish 2006). Heart rate variability becomes more regular with parasympathetic domination after meditation, signifying a health benefit for persons under stress conditions (Wu \& Lo 2008). 

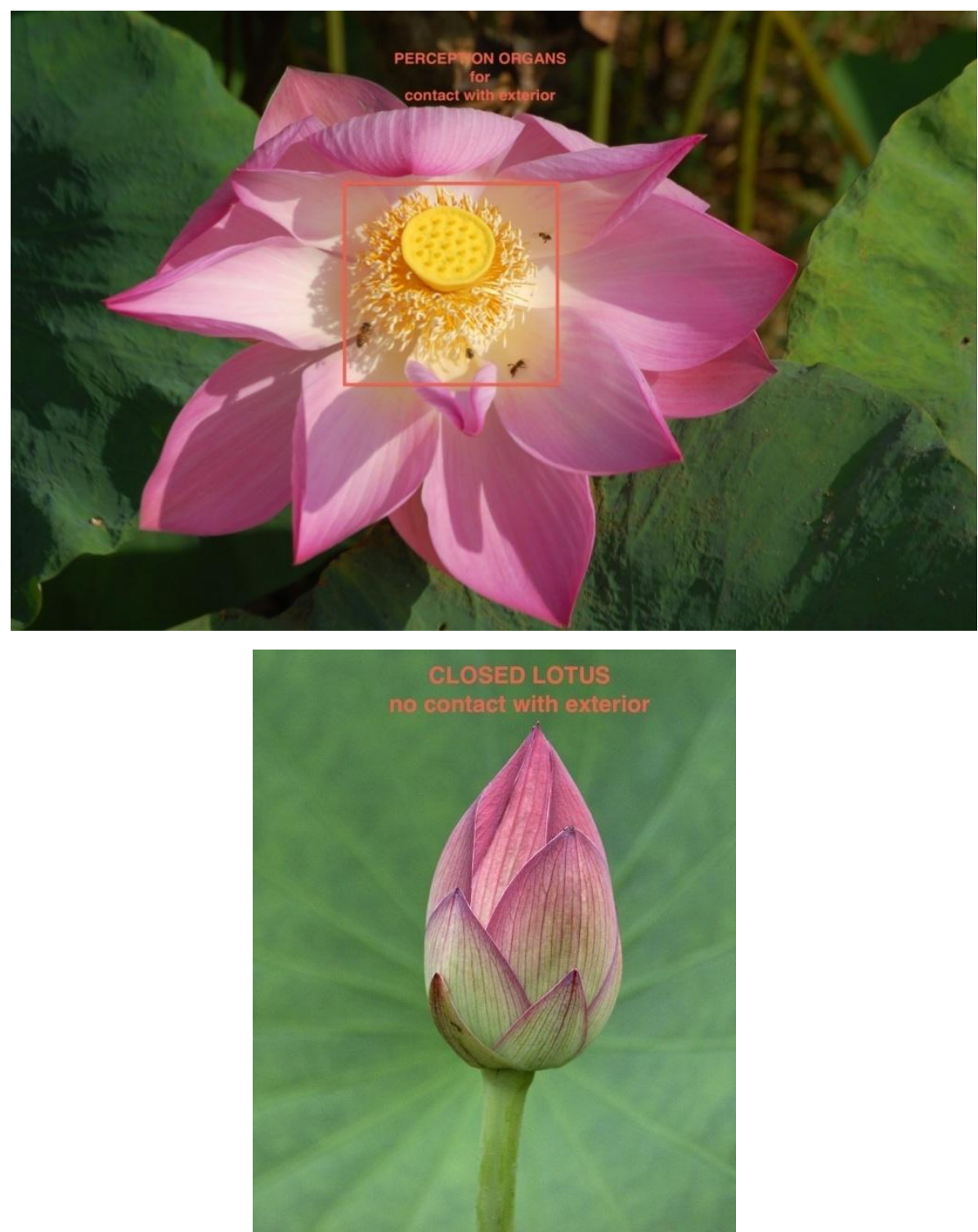

Fig. 2 and Fig. 3 Opening and closing of sensory organs symbolised by the opening and closing of a lotus flower. In the open state (Fig. 2), the flower is in contact with insects from the outside environment, like a duality state, and in the closed state (Fig. 3), the contact is disrupted representing a non-duality state.

In clinical studies, meditation induces a modest lowering of blood pressure and is recommended for the treatment of increased blood pressure (Brook et al. 2013). During the treatment of psoriases, mindfulness meditation reduces stress during phototherapy and thereby increases the rate of resolution of psoriatic lesions and diminishes the risk of skin cancer (Kabat-Zinn et al. 1998). In cancer therapy, meditation induces behavioural and neurophysiological changes alleviating cancer related impairments, thereby increasing quality of life (Kelly et al. 2009).

Changes in gene expression are found in the more recent literature. Transcriptional profiles show that peripheral blood leucocytes reduce the pro-inflammatory nuclear factor (NF) and increase anti-viral interferon responses. The expression of 68 genes is changed, most with down and some with up regulation (Black et al. 2012). In peripheral blood monocyte cells, mindfulness meditation reduces the expression of histone 
deacetylase genes and the expression of inflammatory genes suggesting a potential for the treatment of chronic inflammatory conditions (Kaliman et al. 2014). After intensive meditation with training over three months, telomerase activity significantly increases as compared to controls, which increases cellular lifetime (Epel et al. 2009).

\subsection{Convergence With Quantum Mechanical Theories}

The experience of timeless and spaceless, non-dual consciousness resembles some characteristics of quantum mechanics (Kaufman 2014), in which elementary particles cannot be observed with certainty due to Heisenberg's uncertainty principle. Some characteristics of quantum mechanics proposed by physicists are:

1. superposition of wave functions, which collapse when subjectively observed;

2. wave-particle complementarity needing a subjective choice for experimentation;

3. non-locality with interaction at distance, superior to the speed of light;

4. entanglement of all interactions indicating wholeness of the entire system.

The mathematical formalism with timelessness, spacelessness, and non-locality is explained by several quantum mechanical interpretations. Schrödinger's wave function considers elementary particles to be in superposition, which is unobservable and may or may not collapse to observable classical states. According to the Copenhagen interpretation, superposition has to collapse. This can also be explained with the decoherence theory by interaction with the environment (Zurek 1981). Under the opposite hypothesis that they remain in superposition, Everett (1957) proposes the simultaneous existence of parallel worlds.

Kafatos and others (2011) describe the consequences of quantum mechanics, when they are applied to brain dynamics, which acquire quantum states like the atoms of which they are composed. "The interconnectedness of everything is particularly evident in the non-local interactions of the quantum universe." It follows that "Consciousness and matter are not fundamentally distinct but rather are two complementary aspects of one reality..." (Kafatos et al. 2011, 2). Western science is essentially based on the subject-object dichotomy, but the theory of quantum mechanics allows unifying two apparently different realities into one, thereby leading to "the disappearance of the material universe implied by quantum theory" (Kafatos et al. 2011, 4).

Penrose (1994) suggests a different theory also depending on the collapse of superposition. Non-computable quantum gravity thresholds in space-time geometry could induce the collapse of superposition in an isolated quantum system. Hameroff and Penrose (1996) adapt this theory to biology and propose that the protein tubulin, which is associated in long strains to microtubules in nerve cells, could be the support for quantum superposition. Tubulin can switch between two conformational states and the electrons responsible for the corresponding dipoles are considered in superposition (simultaneously in two states), thereby also inducing superposition of two conformational states of the protein. Due to non-computable objective factors, the system can collapse by objective reduction (OR) to the classical conformation. In contrast to random decoherence (Zurek 1981), OR is orchestrated by physical and biological factors and called Orch OR. With a pan-psychist view consistent with modern physics, this phenomenon is thought to influence proto-consciousness, able to induce qualia experiences in consciousness. "... modern pan-psychists attribute protoconscious experience to a fundamental property of physical reality" (Hameroff 1998, 1871). The western view of panpsychism evokes some similarity, although not identity with the eastern philosophy on pure consciousness. 


\section{Divergent Explanations of the Subjective Experience of Consciousness}

Although there seems to be convergence between eastern philosophy and western science on the existence of the subjective experience of consciousness during the practice of profound meditation and on its benefits for personal wellbeing, health, and social behaviour, there is divergence on its explanation. Subjective experiences can sometimes be explained in a different way with objective concepts. The best known classical examples are:

1. the subjective experience of a flat earth, which was considered by the ancient Egyptians as being objectively flat;

2. the daily convincing subjective experience of sunrise in the east and sunset in the west, which was for many centuries interpreted as the sun turning objectively around the earth;

3. the subjective experience of the earth was believed as the centre of the universe until billions of stars were discovered in the milky way galaxy, which are also surrounded by billions of other galaxies.

Thus, convincing subjective experiences may have different objective explanations, which could nevertheless explain the same subjective experience in another way.

In eastern philosophy, the experience of timeless consciousness during profound meditation is explained as a proof for the existence of pure consciousness, a spiritual entity, which is irreducible to material forms and existed before the material world (Rao 2005). This impersonal spiritual entity is different from a personalised God, believed by most western religions. Chopra characterises spirituality as "an impersonal God who permeates every atom of the universe and every fibre of our being" (Chopra \& Molinow 2011, 7). However, from a western scientific viewpoint, the concept of consciousness includes both, timeless and time dependent experience of consciousness as a whole, in which timeless consciousness with partially inactive sense organs is only a special manifestation of time dependent consciousness with active sense organs.

\subsection{Different Interpretations of the Experience of Consciousness}

Eastern philosophy clearly separates the subjective timeless experience of consciousness with partially inactive sense organs during deep meditation from all conscious experiences of the body and its environment with fully active sense organs. Meditation allows the participation in pure consciousness. "Participating in pure consciousness is a way of knowing in being" (Rao 2005, 8). Pure consciousness is a spiritual entity, but accessible by the human mind, which "has the capacity to interface with consciousness at one end and the brain at the other... the person is embodied consciousness... consciousness is fundamental and irreducible to brain states" (Rao 2005, 9).

The experience of consciousness in deep meditation testifies a participation of the spirit of pure consciousness.

Purusha (spirit) is pure consciousness. It is the transcendental principle underlying all knowledge. In the embodied person with its proximity to purusha, there is the phenomenal experience of unity, coherence, continuity, and selfhood... By an association with the purusha, the mind... becomes conscious... manifests subjectivity and has phenomenal awareness. (Rao $2005,11)$

The undeniable experience of timeless consciousness in deep meditation is explained as an empirical proof for the existence of pure consciousness, a spiritual entity. “... the claims of reality of the states of pure consciousness are not confined to metaphysical presuppositions, but are said to be grounded in genuine experiences of a few people" (Rao 2005, 17). 
For western science, there is no doubt on the existence of the subjective experience of consciousness in deep meditation, however, the explanation that this experience is the proof of a spiritual entity of pure consciousness is only considered as an interpretation, which seems to follow a kind of an anthropic principle, suggesting that pure consciousness allows the final accomplishment of an individual person. "Experience of pure consciousness is considered to be a life-transforming experience" (Rao 2005, 22). It has to "liberate the person from this predicament and lead the individual to higher states of awareness, achievement, and enjoyment... and thus overcome suffering" (Rao 2005, 10).

From a western scientific viewpoint, time dependent and timeless experiences of consciousness remain neutral with respect to an anthropic principle. Both kinds of experiences are considered as the expression of the same consciousness, which can by deep concentration find the subjectivity of timeless, non-dual consciousness experience, but also again experience objective time dependent dualities by expanding to the environment.

\subsection{Time Limitation of the Experience of a Timeless Consciousness}

In eastern philosophy, the experience of pure consciousness is an ideal situation, where in the higher states of awareness all suffering disappears. Thus, the higher awareness of pure consciousness should be the ideal state to obtain by each individual. However, there is a major time limitation, since the experience of pure consciousness cannot be extended over more than some hours per day. Buddhist monks also interrupt their meditation in order to ask for food in surrounding villages, as required for the survival of their body. At the end of meditation, all favourable conditions allowing the timeless experience of consciousness are diminished and unfavourable time dependent conditions, like weather conditions and social interferences, have to be confronted again. Thus, the ideal situation for overcoming all suffering and reaching higher states of awareness is regularly interrupted and could not extend to the whole day or month or even whole life. The interruption of meditation is imposed by the body and its need for daily food uptake, muscle movements, and sleep for survival. Thus, with a scientific western view, time limitation of experiencing deep consciousness indicates the interdependence of two realities, profoundly depending on each other. The body needs time dependent consciousness to master daily environmental problems and the experience of timeless consciousness in deep meditation helps overcome stress situations, but requires a healthy body with a functioning brain.

\subsection{Creation of the Universe by Design or Selection}

The eastern view of pure consciousness designing and creating the universe is in conflict with the western viewpoint of Darwinian selection in biology. Deepak Chopra explains:

A Swiss watch does not just pile a bunch of simple processes on top of one another, it has purpose and meaning. It was designed to perform a specific task... All these aspects of design must have come from somewhere. Spirituality argues that they are aspects of consciousness, the invisible designer behind the scenes of the visible world. (Chopra \& Mlodinow 2011, 118)

The prior design of the world is in opposition to random creation as envisaged by western science. There might first be a major semantic problem, since randomness can be interpreted in two opposite ways. It could mean ontological indeterminism, in which there are no causal chains of events only probabilities, but it can also be interpreted as epistemic indeterminism, in which there are still determinist causal chains of events, but our knowledge is too limited for knowing them. The philosopher Dennett explained with a coin toss, as an example of indeterminism, that the "result of a coin flip is the deterministic outcome of the total sum of forces acting on 
the coin" (Dennett 2004, 85). The uncertain outcome of the coin flip is only epistemic, since it cannot be predicted due to the high complexity of all interfering factors. Nevertheless, at the ontological level, there are multiple determinist forces acting on the coin toss and, if one could evaluate each of them, the outcome would become predictable. Thus, at the epistemic level, randomness is not considered non-causal, but only indicates that we have no knowledge on the complexity of underling causal chains, although they really exist.

The main point distinguishing eastern philosophy from western science concerns the design of the universe by an impersonal power, like pure consciousness, which is opposed to a progressive Darwinian evolution by selection with determinist causes. Designing as well as selecting is typically human processes and their underlying principles can be considered for explaining the creation of the universe. Designing requires two conditions, prior knowledge, how the designed factors will proceed and homogeneity of the designed factors to become effective. At the human level, knowledge has first to be acquired individually or transmitted by the society and then allows designing. However, designing is impossible under random conditions. A gold searcher cannot design, when and where he will find gold and a fisherman cannot design when and what kind of fish he will bring home. Homogeneity of the starting material is essential and created by human civilisations during centuries of evolution. Only the extraction of pure iron from heterogeneous ferruginous rocks allowed the design of iron tools. If the starting conditions are heterogeneous at the beginning, they can nevertheless become homogeneous by a selection process. The Darwinian Theory on the evolution of new species is based on selection by the environment from random genetic material. Thereafter, homogeneous conditions can be maintained and allow proliferation of the selected homogeneous material.

Any action programmed by human intelligence requires prior acquired knowledge, since without any knowledge, a person is limited to "observing and wondering." With incomplete knowledge due to random conditions or inhomogeneous materials, a person can proceed to be "expecting and selecting," but only complete knowledge based on homogeneous conditions and homogeneous materials allow "knowing and designing." The possibilities of human intelligence can be projected on the creation of the universe. Eastern philosophy attributes the ideal conditions of complete knowledge to pure consciousness for designing, in a similar way as western religions consider a personalised God. Only western science conceives the evolution of the universe from inhomogeneous starting conditions by evolution and selection.

\subsection{Compatibility of a Design With Error or Evil}

A human design affected under optimal conditions, with complete knowledge and homogeneous starting conditions and materials should realise a flawless designed entity. There should be no reason that a design under ideal conditions by pure consciousness should include errors, which would require incomplete knowledge or inhomogeneous starting conditions and materials.

Although eastern philosophy attributes the universe to a design by pure consciousness, human life conditions reveal major errors in the design. Such errors, not due to human intervention, are experienced as evil and injustice, which is encountered in all populations of the world. Unavoidable evil is found in birth defects, which occur in about 3\% of new born children (WHO, 2014a). Congenital heart diseases are about $1 \%$ (Linde et al. 2011), and craniofacial birth defects are in the order of $0.2 \%$ (WHO, 2014b). More impressive birth defects are found in children born with limb anomalies (dysmelia) in about $0.07 \%$ (Chen 2006), some of which without arms or legs. Hermaphrodite babies with sexual characteristics of both man and female are found in about $0.05 \%$ (Wiki answers). Even more impressive is Siamese twins occurring in about 1/100,000 births 
(Mutchinick et al. 2011), 50\% of which are stillborn. Only about 25\% of siamese twins can survive under difficult conditions (Wikipedia, conjoined twins).

Besides visible birth defects, future social behaviour seems also to be genetically programmed (Barnes et al. 2011), since psychopathic criminals are found to have brain defects in the paralimbic gray matter including the amygdala and hippocampal regions (Ermer et al. 2012). Imaging brain studies show that psychopathics criminals feel less empathy than controls (Meffert et al. 2013). The interpretation in eastern philosophy of an ideal design of the universe by pure consciousness is difficult to conceive with major errors, such as $3 \%$ birth defects and genetically transmitted psychopathic brain defects favouring criminality.

Western religions have the same problem of explaining an omnipotent God and the simultaneous presence of good and evil in the world. In the ancient Greek mythology, good and evil are not yet separated from God, since Zeus decides on both. "Everything good as well as bad comes from Zeus, and according to his own choice, he assigns their good or evil lot to mortals" (Smith 1870, 1322). In later monotheist religions, good and evil are separated so that God only represents the good, which needs a demon for the evil. In the christian religion, "Evil appeared in the world of angels when Lucifer, one of God's cherubs, rebelled against this order" (Valea 2014). In Islam, Alah is the representation of the good and Shairan (Satan) is the representation of the evil, therefore, "each individual is accompanied by two personal spiritual entities; an angel who urges toward good and a shairan who urges toward evil" (Bowker 1997).

In eastern philosophy according to Feuerstein (2003), the manifold universe is a single reality with one great being, called pure consciousness, which is the essence or self of all beings. Rao writes: "When awareness moves to the level of pure consciousness... subject-object duality experienced at the empirical level disappears" (Rao 2005, 17). "In the embodied person with its proximity to purusha (spirit), there is the phenomenal experience of unity, coherence, continuity, and selfhood" (Rao 2005, 11). In Hinduism, the concept of advaita (non-dualism) during the experience of pure consciousness does not admit the opposite forces of good and evil. Thus, the evil of birth defects does not disappear and is only hidden during the experience of consciousness, but reappears at the end of meditation, when extra-mental reality with active sense organs is reintegrated in the duality world again.

Any human design corresponds to human creation after acquired knowledge. Whereas human creations with $97 \%$ flawless realisations would be considered as a great success, $3 \%$ errors after an ideal design by pure consciousness are difficult to accept. However, creation by mutation and selection could be more adapted for explaining the $3 \%$ errors after continuous evolution of multiple species appearing and disappearing during the last billion years.

\section{Conclusion}

Consciousness is very difficult to explain, since it includes the mind-body problem concerning the coexistence of subjectivity and objectivity, which profoundly divides eastern philosophy and western science. In normal life, consciousness is a time dependent experience, but during deep meditation, it reveals a special timeless character. In eastern and western countries, meditation has become a widely accepted practice with encouraging effects on psychological and biological wellbeing. These effects are scientifically well documented including neuro-scientific experiments. Nevertheless, the interpretation of the experience of consciousness is diverging between eastern philosophy and western science.

A pure phenomenological analysis of consciousness reveals two opposite experiences, a time dependent 
experience of consciousness, when all sense organs are active and open to environmental stimuli, and an experience of non-dual timeless consciousness during profound meditation after neutralisation of many sense organs. There is divergence for the interpretation of the subjective experience of timeless consciousness in higher order awareness, which is in eastern philosophy considered as the embodiment of pure consciousness in a person, a spiritual force existing before the material world and creating it by design. Therefore, time dependent consciousness has only a secondary role for perception and cognition, but the experience of timeless consciousness in meditation enables a more enjoyable life by calming general stress and diminishing disease dispositions. In contrast, western science considers time dependent and timeless consciousness as a whole with equivalent values, in which time dependent consciousness essentially maintains the body in health, thereby allowing the experience of timeless consciousness during meditation.

Eastern pure consciousness is thought to design the universe. Designing is a characteristic human action, which depends on prior knowledge and homogeneous conditions. Without any knowledge, no human action is possible, only passive "observing and wondering." With incomplete knowledge due to inhomogeneous conditions, there is only "expecting and selecting," whereas "knowing and designing" require prior complete knowledge and homogeneous conditions. Inspired by the human capacities, there are two possibilities for explaining the creation of the universe: the eastern philosophical view of creating constant entities by design under homogeneous conditions or the western scientific view of constantly evolving diversities needing selection due to inhomogeneous starting conditions. The eastern non-dual concept of pure consciousness cannot accept selection as a creation process, since it would require the pre-existence of the material world before the spiritual universe. The western viewpoint considers the time dependent and the timeless consciousness as equivalent and profoundly interdependent. Meditation cannot become permanent and has regularly to be interrupted for following the requirements of the body. Thus, timeless consciousness could not be experienced without the time dependent consciousness essential for the survival of the body. The western concept is in agreement with selection under inhomogeneous conditions and tolerates the duality of good and evil. The fundamental injustice of $3 \%$ congenital abnormalities is not in disagreement with western science, but more difficult to explain with an ideal design by pure consciousness. Eastern philosophy has the advantage of rendering normal life more enjoyable and eliminating suffering by meditation, although only for a limited time period, whereas western science can try to eliminate congenital injustice after investigation and sometimes reparation of the causal factors by surgery.

The analysis of consciousness by eastern philosophy and western science is partially convergent and partially divergent. Chopra writes in favour of the eastern viewpoint that "mechanistic determinism offers no personal satisfaction and has... to face a universe that is cold and void..." (Chopra and Mloninow 2011, 261). Accordingly, there is a general expectation for finding comprehension, creation and love, but why has this to be imposed by pure consciousness in the universe when claiming "... the universe is also loving through you, creating through you and evolving through you..." (Chopra and Mloninow 2011, 229)? Humans themselves create environments with comprehension and love, but also tolerance with respect to other mentalities. Spirituality (Chopra Centre) as well as all great religions and charitable organisations practise comprehension and tolerance continuously in their communities.

In summary, eastern philosophy and western science show a certain degree of complementary, the practice of eastern meditation leads to better subjective and objective wellbeing and the practice of western scientific investigation of illness can sometimes eliminate objective injustice and thereby subjective suffering. 


\section{Works Cited}

Barnes, J. C., Beaver, Kevin, and Boutwell, Brian. "Examining the Genetic Underpinnings to Moffitt's Developmental Taxonomy: A Behavior Genetic Analysis.” Criminology 49.4 (2011): 923.

Biegler, Kelly, Chaoul, Alejandro, and Cohen, Lorenzo. Cancer. "Cognitive Impairment and Meditation." Acta Oncologica 48 (2009): 18-26.

Black, D. S., Cole, S. W., Irwin, M. R., Breen, E., St Cyr, N. M., et al.. "Yogic Meditation Reverses NF-kappaB and IRF-related Transcriptomte Dynamics in Leukocytes of Family Dementia Caregivers in a Randomised Controlled Trial." Psychoneuroendocrinology 38.3 (2012): 348-55.

Bowker, John. The Oxford Dictionary of World Religions. New York: Oxford University Press, 1997. 273.

Brook, Robert, and 11 colleagues. "Beyond Medications and Diet: Alternative Approaches to Lowering Blood Pressure: A Scientific Statement From the American Heart Association." Hypertension 61 (2013): 1360-83.

Cahn, Rael and Polish, John. "Meditation States and Traits: EEG, ERP, and Neuroimaging Studies." Psychological Bulletin 132.2 (2006): 180-211.

Chen, H. FAAP, FACMG. “Dysmelia.” Atlas of Genetic Diagnosis and Counselling. Berlin: Springer, 2006. 312-22.

Chopra, Deepak and Mlodinow, Leonard. "Is There a Design in the Universe." War of the Worldviews. Science Versus Spirituality. New York: Harmony Books, Crown Publishing Group, 2011. 107-121.

Dennett, Daniel. Freedom Evolves. New York: Penguin Books, 2004.

Eagleman, David and Pariyadath, Vani. "Is Subjective Duration a Signature of Coding Efficiency?" Phil. Trans. R. Soc. B 364 (2009): 1841-51.

Epel, Elissa, Daubenmier, J., Moskowitz, J. M., Folkman, S., and Blackburn, E.. "Can Meditation Slow Rate of Cellular Aging? Cognitive Stress, Mindfulness, and Telomeres.” Ann N Y Acad. Sci. 1172 (2009): 34-53.

Ermer, E., Cope, L. M., Nyalakanti, P. K., Calhoun, V. D., and Kiehl, K. A.. "Aberrant Paralimbic Gray Matter in Criminal Psychopathy." Journal of Abnormal Psychology 121.3 (2012): 649-58.

Everett, Hugh. "Relative State Formulation of Quantum Mechanics." Quantum Theory and Measurement. J. A., Wheeler and W. H., Zurek, eds.. Rev. Mod. Physics 29 (1957): 454-62.

Feuerstein, Georg. The Deeper Dimension of Yoga: Theory and Practice. Boston: Shambhala Publications, 2003.

Hameroff, Stuart and Penrose, Roger. "Orchestrated Reduction of Quantum Coherence in Brain Microtubules: A Model for Consciousness." Toward a Science of Consciousness. I. The first Tucson Discussions and Debates. Ed., Stuart, Hameroff, A., Kaszniak, and A. C., Scott. Cambridge: MIT Press, 1996. 507-40.

Hameroff, Stuart. "Quantum Computation in Brain Microtubules? The Penrose-Hameroff 'Orch OR' Model of Consciousness.” Phil. Trans. R. Soc. Lond. A 356 (1998): 1869-96.

Jansen, Franz Klaus. "Oscillation Between Time and Timelessness Corresponding to Duality and Non-duality Perception." Proceedings of the Science and Non-duality Conference. St. Raphael, CA, 2011. 49.

Jansen, Franz Klaus. "The Observer's Now, Past and Future in Physics From a Psycho- Biological Perspective." Cosmology 18 (2014): 376-401. <http://cosmology.com/ConsciousTime112.html>.

Kabat-Zinn, J., Wheeler, E., Light, T., Skillings, A., Scharf, M. J., Cropley, T. G., Hosmer, D., and Bernhard, J. D.. "Influence of a Mindfulness Meditation-Based Stress Reduction Intervention on Rates of Skin Clearing in Patients With Moderate to Severe Psoriasis Undergoing Phototherapy (UVB) and Photochemotherapy (PUVA).” Psychosomatic Medicine 60 (1998): 625-32.

Kafatos, Menas, Tanzi, Rudoph E., and Chopra, Deepak. "How Consciousness Becomes the Physical Universe." Journal of Cosmology 14 (2011).

Kaliman, P., Álvarez-López, M. J., Cosín-Tomás, M., Rosenkranz, M. A., Lutz, A., and Davidson, R. J.. "Rapid Changes in Histone Deacetylases and Inflammatory Gene Expression in Expert Meditators.” Psychoneuroendocrinology 40 (2014): 96.

Kaufman, Steven. "The Nature of Quantum Reality: What the Phenomena at the Heart of Quantum Theory Reveal About the Nature of Reality (Part III)." Journal of Consciousness Exploration \& Research 5.1 (2014): 65-84.

Kelly, A., Biegler, M., Chaoul, A., and Cohen, L. Cancer. “Cognitive Impairment and Meditation.” Acta Oncologica 48 (2009): 18-26.

Linde, D. van, Konings, E. E. M., Slager, M. A., Witsenburg, M., Helbing, W. A., Takkenberg, J. J. M., and Roos-Hesselink, J. W.. "Birth Prevalence of Congenital Heart Disease Worldwide. A Systematic Review and Meta-Analysis." JACC 58.21 (2011): 2241-2247. 
Meffert, H., Gazzola, V., den Boer, J. A., Bartels, A. A. J., and Keysers, C.. "Reduced Spontaneous but Relatively Normal Deliberate Vicarious Representations in Psychopathy." Brain 136.8 (2013): 2550.

Mutchinick, O. M.. "Conjoined Twins: A Worldwide Collaborative Epidemiological Study of the International Clearinghouse for Birth Defects Surveillance and Research.” Am J Med Genet C Semin Med Genet 15 (2011): 274-87.

Penrose, Roger. Shadows of the Mind. Oxford: Oxford University Press, 1994.

Rao, K. R.. "Perception, Cognition and Consciousness in Classical Hindu Psychology.” J. Consciousness Studies 12.3 (2005): 3-30.

Smith, William. "Zeus.” Dictionary of Greek and Roman Biography and Mythology. Boston: Little, Brown and Company, 1870. 1322.

Valea, E.. "Comparative Religion-The Problem of Evil in World Religions (2014)." <http://www.comparativereligion.com/evil.html\#Christianity>.

Vimal, Ram, L. Pandy. 'Meanings Attributed to the Term 'Consciousness:' An Overview.” J. Consciousness Studies 16.5 (2009): 9-27.

WHO. “Congenital Anomalies." Fact Sheet No. 370. (2014a) <http://www.who.int/mediacentre/factsheets/fs370/en/>.

WHO. "Human Genetics.” Craniofacial Abnormalities. (2014 b) <http://www.who.int/genomics/anomalies/en/>.

<wiki.answers.com/Q/User: Hippydog>.

<http://www.answers.com/Q/What_percentage_of_babies_born_in_the_US_are_hermaphrodites_having_sexual_characterictics_o f_both_male_and_female $>$.

Wu, S. D., and Lo, P. C.. "Inward-attention Meditation Increases Parasympathetic Activity: A Study Based on Heart Rate Variability." Biomedical Research 29.5 (2008): 245-50.

Zurek, Wojciech. "Pointer Basis of Quantum Apparatus: Into What Mixture Does the Wave Packet Collapse?" Phys Rev D24 (1981): 1516-25. 